\title{
Effectiveness of quadrivalent influenza vaccination in the first year of a funded childhood program in Queensland, Australia, 2018
}

DOI: $\underline{\text { https://doi.org/10.1016/j.vaccine.2020.12.012 }}$

\section{Authors and affiliations}

Dharshi Thangarajah ${ }^{\mathrm{a}, \mathrm{b}}$, Jonathan A. Malo ${ }^{\mathrm{a}}$, Emma Field ${ }^{\mathrm{b}, \mathrm{c}}$, Ross Andrews ${ }^{\mathrm{b}, \mathrm{c}}$, Robert S. Ware ${ }^{\mathrm{d}}$, Stephen B. Lambert ${ }^{a, b}$

${ }^{a}$ Communicable Diseases Branch, Queensland Health, Brisbane, Australia

${ }^{\mathrm{b}}$ National Centre for Epidemiology and Population Health, Australian National University, Canberra Australia

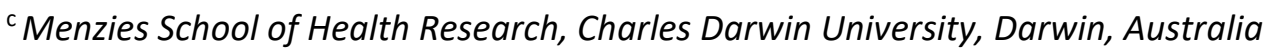

${ }^{\mathrm{d}}$ Menzies Health Institute Queensland, Griffith University, Brisbane, Australia

\section{Corresponding author}

Ms Dharshi Thangarajah

dharshi.thangarajah@anu.edu.au 


\section{Abstract}

Background: Following high influenza activity in 2017, the state of Queensland, Australia, funded a quadrivalent inactivated influenza vaccination program for children aged 6 months to $<5$ years in 2018 . We calculated influenza vaccine effectiveness (VE) among children eligible for this program.

Methods: A matched case-control study was conducted. Cases were identified using Queensland 2018 influenza notification data among children age-eligible for funded vaccination. Controls were drawn from Australian Immunisation Register records of Queensland resident children age-eligible for funded influenza vaccine. Up to 10 controls per case were matched for location and birthdate. First dose vaccination was valid if received $\geq 14$ days prior to specimen collection; a second dose was valid if received $\geq 28$ days after first dose receipt. VE was calculated for vaccine doses and adherence to national recommendations for two doses in the first season (schedule completeness) and adjusted $\left(V E_{a d j}\right)$ for sex and First Nations status.

Results: There were 1,125 cases and 10,645 matched controls analysed. Overall $V E_{a d j}$ against laboratory-confirmed influenza was 51\% (95\% confidence interval (Cl) $41-60)$. VEadj was $60 \%(95 \% \mathrm{Cl}$ $46-70)$ for children who received two doses in 2018 , and $60 \%$ (95\% Cl 48-69) for children vaccinated appropriately according to schedule completeness. VE increased with age.

Conclusions: Moderate vaccine effectiveness was observed for children eligible for the funded program in Queensland in 2018, adding to the sparse evidence for influenza vaccine use in Australian children. Adhering to the national first season two dose schedule for influenza vaccine receipt in children ensures maximum protection.

\section{Keywords}

influenza; influenza vaccine; vaccine effectiveness; children; Australia 


\section{Background}

Influenza is the most commonly notified vaccine preventable disease in Australia [1, 2]. Children, older adults, and those with weakened immune systems, are most vulnerable to influenza infection. Children experience greater influenza severity as evidenced by consistently high hospitalisation rates in this age group [3].

Population-based influenza vaccination programs reduce the risk of influenza infection and its associated morbidity through direct protection, and indirectly through herd protection [4]. Models, observational, and experimental studies show that children play a key role in transmission of influenza to their peers and other age groups $[5,6]$. Cessation of a nation-wide mass immunisation program of school children in Japan saw a rise in excess population mortality rates attributed to pneumonia and influenza [7]. A randomised trial in Canada demonstrated that vaccinating children produced substantial indirect protection against influenza in unvaccinated people in other age groups [8]. Vaccinating children against influenza is important in not only reducing the risk of influenza infection and disease severity in children, but also in preventing transmission to other high-risk populations [9].

In 2017, Australia experienced the highest influenza activity on record, with 251,289 laboratoryconfirmed cases reported nationally (annual notification rate: 1,022 per 100,000 population per year) [2]. The state of Queensland (a tropical/subtropical region) had Australia's third highest notification rate $(1,149$ per 100,000 population per year), with a total of 56,614 influenza cases notified [2]. This absolute number of notifications was 2.5 times greater than the mean annual number of influenza notifications from 2014-2016 [2]. In 2017, children <1 year of age experienced the highest rate of laboratory-confirmed influenza admissions to Queensland public hospitals (292 per 1,000 notifications) when compared to other age groups [10].

In response to the 2017 flu season, seven states and territories, including Queensland, funded influenza vaccination programs from 2018 for children aged 6 months to $<5$ years old $[11,12]$. Under 
the funded program in Queensland, children in this age group are eligible to receive free quadrivalent influenza vaccination (QIV). Influenza strains included in the 2018 southern hemisphere QIV were: A/Michigan/45/2015 (H1N1)pdm09-like virus, A/Singapore/INFIMH-16-0019/2016(H3N2)-like virus, B/Phuket/3073/2013-like virus, and B/Brisbane/60/2008-like virus [12]. QIV brands registered for use in children in Australia in 2018, and distributed in Queensland under the funded program were: FluQuadri Junior (Sanofi Pasteur) for children 6 months to $<3$ years of age; and FluQuadri (Sanofi Pasteur) and Fluarix Tetra (GSK) for children $\geq 3$ years to $<18$ years of age [12]. The Australian Immunisation Handbook recommends that children aged 6 months to $<9$ years receive two vaccine doses (at least 4 weeks apart) in their first year of receiving influenza vaccine, followed by one dose in subsequent years [13].

Measuring vaccine effectiveness (VE) provides evidence for how well vaccines work to protect against influenza in the context of a population vaccination program. To date, estimates of influenza VE in Queensland children have not been calculated. The primary objective of this study was to use routinely collected data to assess vaccine effectiveness in preventing laboratory-confirmed influenza infection in eligible children during the first year of implementation of the publicly funded childhood influenza vaccination program in Queensland.

\section{Methods}

\subsection{Study design}


For the analysis of VE against laboratory-confirmed influenza in this study we undertook a matched case-control study.

\subsection{Cases}

Laboratory-confirmed influenza has been a notifiable condition in Queensland since 2001. The Queensland Public Health Act 2005 and its subordinate regulation requires public and private Queensland laboratories to report all cases of influenza meeting at least one of the following influenza laboratory notification criteria to Queensland's Notifiable Conditions System (NoCS) $[14,15]$ : isolation of influenza virus by culture from an appropriate respiratory tract specimen; detection of influenza virus by nucleic acid test (NAT) from an appropriate respiratory tract specimen; detection of influenza antigen from an appropriate respiratory tract specimen; IgG seroconversion or a significant increase in antibody level or a fourfold or greater rise in titre to influenza virus; single high titre IgA to influenza virus [16]. Laboratory notifications are primarily reported to the surveillance system electronically. To ensure reporting completeness, Queensland Health regularly engages with notifying laboratories and routinely undertakes routine data quality improvement activities, including disease-specific audits. The Queensland laboratory notification criteria align with the national seasonal influenza case definition for reporting to the National Notifiable Diseases Surveillance System (NNDSS) [17]. Queensland residents diagnosed with influenza in other Australian jurisdictions are notified to Queensland's influenza surveillance system in accordance with the national Cross-Border NNDSS Notification Protocol [18].

In this study, cases were children age-eligible for funded vaccination notified with laboratoryconfirmed influenza reported to NoCS in 2018. Cases were excluded if they were identified as having dual influenza strain infection. Case data were extracted from NoCS on 23 September 2019.

Publicly funded influenza vaccines were distributed in Queensland from 10 April 2018 (personal communication, Vicki Bryant, Clinical Nurse Consultant, Queensland Immunisation Program). To allow 
for vaccine distribution and the 14 days required to confer any active immunity from a vaccine, cases were restricted to children aged $\geq 6$ months and 14 days ( $\geq 196$ days) at the time of specimen collection, with a specimen collection date from 01 May 2018 to 31 December 2018. This gave cases included in this study a date of birth range from 15 April 2013 to 15 June 2018.

\subsection{Matched controls}

The Australian Immunisation Register (AIR) is a whole-of-life national vaccination register [19]. Deidentified data for potential controls were obtained from AIR records of all Queensland-resident children born from 15 April 2013 to 15 June 2018.

Statistical area 2 (SA2) regions are Australian Statistical Geography Standard structures used to coalesce communities that interact together socially and economically [20]. SA2 areas range in population from 3,000 to 25,000 people (average: approximately 10,000 people) [20]. SA2 (2016) values for cases and controls were derived from their recorded postcode of residence using Australian Statistical Geography Standard (ASGS) postcode to SA2 correspondence files [21].

Controls were matched to cases on SA2 and a date of birth \pm 14 days from the birthdate of the case. Cases not able to be matched to any controls on SA2 and \pm 14 days from case birthdate criteria were excluded from the analysis.

Controls with the same birthdate and sex as their matched case were excluded to avoid the possibility of a case acting as their own control. Where a control matched to two or more cases, the control was randomly assigned to a single case only. The number of controls matched to a case was restricted to 10. Where $>10$ controls matched to a single case, 10 controls were randomly selected from all possible controls for that case using computer generated random numbers. To ensure controls were of the same dose age-eligibility as matched cases, controls were excluded if aged $<6$ months and 14 days ( $<196$ days) at specimen collection date for matched cases who received no or one eligible dose, or if 
aged $<7$ months 14 days (<226 days) at specimen collection date for matched cases who received two eligible doses.

\subsection{Vaccination status}

The 2018 influenza vaccination status for cases was validated using the AIR as part of routine public health activity. Cases were excluded if their vaccination status was unable to be validated (i.e. case was not located in AIR records). For controls, influenza vaccinations received in 2018 were provided in their de-identified AIR record. Control AIR data for this project were extracted on 10 September 2019.

An influenza vaccination received in 2018 was considered valid if received $\geq 14$ days prior to the specimen collection date of the case (for controls, the specimen collection date of the matched case). A second dose was considered valid if received $\geq 28$ days after receipt of the first valid 2018 influenza dose and $\geq 14$ days prior to the specimen collection date of the case. Where a second dose was received $<28$ days after receipt of a first valid dose, the child was considered to be a single dose recipient.

The overall influenza vaccine dose number received in the child's lifetime was available for cases and controls who received any influenza vaccine in 2018. Children were categorised for 2018 valid influenza vaccine doses received as follows: no vaccination in 2018; one dose in 2018 and at least one dose received prior to 2018; one dose in 2018 and no dose received prior to 2018; two doses in 2018 (regardless of doses received prior to 2018). In this paper we refer to case or control adherence to first year recommendations for receipt of vaccine as per national guidelines as being either complete or incomplete. Individuals were classified as complete if they received one dose in 2018 and at least one prior dose, or two doses in 2018; or incomplete if they received one dose in 2018 and no prior dose.

\subsection{Age group}


Case age at the time of specimen collection was categorised as: 6 months and 14 days to $<2$ years; 2 years to $<3$ years; 3 years to $<4$ years; and 4 years to $<6$ years of age. Controls were categorised into the same age groups as their matched case.

\subsection{First Nations status}

The First Nations status of cases and controls was obtained from the NoCS and AIR datasets, respectively. A multiple-category variable for First Nations identification (i.e. Aboriginal; Torres Strait Islander; Aboriginal and/or Torres Strait Islander) was available for cases only, and therefore case and controls were analysed by a binary First Nations/non-First Nations child variable.

\subsection{Remoteness area and Index of Relative Socio-economic Advantage and Disadvantage}

The SA2 of cases and controls was used to derive Index of Relative Socio-economic Advantage and Disadvantage (IRSAD) quintile based on ranking within Queensland and Remoteness Area [22]. The IRSAD score summarises the economic and social conditions of people and households living in an area [23]. There are five categories of remoteness in Australia based on a measure of relative access to services: Major Cities, Inner Regional, Outer Regional, Remote, Very Remote [24].

\subsection{Statistical analysis}

All analyses were conducted as per study protocol. Cases and controls were described by age at specimen collection, sex, First Nations status, IRSAD quintile, remoteness area, 2018 influenza vaccination status, and vaccination completeness. The association between characteristics and casecontrol status was investigated using the chi-squared test. Conditional logistic regression was used to calculate odds ratios (OR). Vaccine effectiveness, against influenza (any) and influenza group and subtype, was calculated as (1-OR) x 100\%. Crude and adjusted estimates of VE (adjusted for sex and First Nations status) were calculated for receipt of any valid influenza vaccine doses in 2018 (yes, no), 
and vaccine dose receipt $(0,1$, or 2 valid doses). Vaccine effectiveness by vaccine completeness in 2018 (no vaccine, incomplete, complete) was stratified by age group. All statistical analyses were conducted using Stata v14.1 (StataCorp, College Station, TX, USA).

\subsection{Ethics approval}

Ethics approval for this study was obtained from the Children's Hospital Queensland Hospital and Health Service Human Research Ethics Committee (LNR/18/QCHQ/43999) and the Australian National University Human Research Ethics Committee (protocol number 2019/44). Release of de-identified line listed AIR data through the National Centre for Immunisation Research and Surveillance was approved by the Australian Government Department of Health. 


\section{Results}

\subsection{Cases and controls}

There were 1,136 cases who met the study eligibility criteria (Figure 1). Eight cases were excluded due to: dual influenza group infection ( $n=2$, both had influenza A:H1N1/H3N2 co-infection), or because their vaccination status was unable to be validated on the AIR $(n=6)$. Three cases were excluded as they did not match to any controls on the required criteria (SA2 and date of birth \pm 14 days from the case's birthdate) leaving 1,125 cases matched to controls. After randomly assigning controls to a case where they were matched to more than one case, controls were randomly selected so that a maximum of 10 controls were matched to each case. Fourteen controls were excluded due to not being ageeligible for the same number of doses as their matched case. A total of 1,125 cases and 10,645 matched controls were included in the study, with $86 \%(n=967)$ of cases matched to 10 controls (Figure 1).

Influenza virus was detected by a nucleic acid amplification test (NAAT) for all 1,125 cases included in the study (NAAT only: $n=1,072$; NAAT and isolation: $n=37$; NAAT and antigen: $n=15$; NAAT, isolation, and antigen: $\mathrm{n}=1$ ). The timing of specimen collection among cases by influenza group, and receipt of influenza vaccine dose among cases and controls (combined) by 2018 calendar week, are presented (Figure 2). Study case notifications peaked in week 36 ( $n=97,8.6 \%$ of cases during study period). Of the 1,125 cases included in the analysis, 1,066 (94.8\%) had Influenza A and 59 (5.2\%) had Influenza B. Subtyping information was available for $17.8 \%(n=190)$ of Influenza A cases: of these, $72.1 \%(n=137)$ were $\mathrm{H} 1 \mathrm{~N} 1$ and $27.9 \%(n=53)$ were H3N2. Lineage was available for only $10.2 \%(n=6)$ of influenza B cases (B/Yamagata: $n=4 ; B /$ Victoria: $n=2$ ), meaning we were unable to assess VE by influenza $B$ lineage.

A total of 2,477 (cases: 143; controls: 2,334) first dose vaccines were received by study participants prior to case influenza specimen collection date, of which $97 \%$ (cases: $94 \%, n=134$; controls: $98 \%$, $n=2,276$ ) were received $\geq 14$ days prior to specimen collection date (valid dose). Timing of receipt of a 
valid first dose of influenza vaccine among study participants during the study period ranged from 07 March 2018 to 13 November 2018, with second doses received from 24 April 2018 to 30 November 2018. Of 58 cases and 1,131 controls where a second dose was recorded, receipt of 29 second doses ( 2 cases and 27 controls) were excluded due to being given $<28$ days following the first dose, and therefore these children were considered single vaccine dose recipients for the purposes of VE analysis. For children who received two valid doses $\geq 14$ days prior to index specimen collection date (54 cases and 1,104 controls), the mean (median) number of days between doses was 37 (39) days. Among study participants, receipt of the first dose peaked in the first week of May 2018 (week 18), with an increase also observed in July 2018 (weeks 27-30). Receipt of the second dose peaked in midJune 2018 (week 24).

The demographic characteristics and influenza vaccination status of cases and controls are summarised (Table 1). As expected, given the matching process, the distribution of age, remoteness area, and IRSAD quintile were similar for cases and controls. Sex was also similar for cases and controls. Compared to other categories, cases were most likely to be aged 4 years to $<6$ years, male, and residents of a major city. Compared to controls, a higher proportion of cases were First Nations children $(p=0.04)$.

\subsection{Influenza vaccine effectiveness}

The overall crude VE for receipt of any influenza vaccine in 2018 was 51\% (95\% confidence interval (CI) 41-59), and remained similar after adjusting for sex and First Nations status (Table 2). When assessing VE by number of doses received, adjusted VE (VEadj) increased to $60 \%(95 \% \mathrm{Cl} 46-70)$ for two dose recipients compared to a $\mathrm{VE}_{\text {adj }}$ of $43 \%(95 \% \mathrm{Cl} 28-55)$ for single dose recipients. The $\mathrm{VE}_{\mathrm{adj}}$ for children who were completely vaccinated according to national guidelines for first year vaccine receipt was $60 \%(95 \% \mathrm{Cl} 48-69)$, and decreased to $35 \%$ (95\% Cl 15-51) for children incompletely vaccinated. When stratified by age group, $\mathrm{VE}_{\mathrm{adj}}$ among those completely vaccinated increased with increasing age group, reaching $77 \%$ and $72 \%$ for children aged 3 to 4 years and 4 to $<6$ years, respectively (Table 3 ). 
Influenza A subtype was not included in the assessment of VE due to a small proportion of influenza A cases where subtype information was available $\left(n=190,17.8 \%\right.$, Table 4). The $V_{\text {adj }}$ against all influenza A was estimated to be $44 \%(95 \% \mathrm{Cl} 28-56)$ for one influenza vaccine dose, and $60 \%(95 \% \mathrm{Cl}$ 46-70) for two doses. For influenza B, point estimates for VE by level of completeness varied, though these values were based on small numbers of cases with wide confidence intervals (Table 4).

\section{Discussion}

During the 2018 influenza season in Queensland, influenza vaccination had a VE of $51 \%$ in preventing any influenza (95\% influenza $A$ in our cases) in children eligible for the publicly funded childhood influenza vaccine in 2018 after adjusting for sex and First Nations status. As expected, compared to those who received one dose in 2018, VE was higher for children who received two influenza vaccine 
doses. This moderate level of protection observed in our study occurred in a year in which circulating influenza $A(H 1 N 1)$ and both influenza $B$ lineages appeared to be antigenically similar to the 2018 southern hemisphere vaccine components [25]. Where able to be assessed, influenza A (H3N2) isolates showed reasonable vaccine-matching [25].

Our VE estimates against laboratory-confirmed influenza are slightly lower than those calculated in two sentinel-site US studies (VE: 60\% against laboratory-confirmed influenza [26]; 68\% against outpatient medically attended influenza [27]) for injectable quadrivalent vaccination in children aged 6 months to $<5$ years during the $2017 / 2018$ northern hemisphere flu season. The components of the northern hemisphere $2017 / 2018$ season vaccine used in the US studies differed by the influenza A H3N2 strain only (northern hemisphere 2017/2018 H3N2 component: A/Hong Kong/ 4801/2014 (H3N2)-like virus; southern hemisphere 2018 H3N2 component used in our study: A/Singapore/INFIMH-16-0019/2016-like virus). Most VE estimates in Australia for children are produced against influenza-related GP presentation [28], hospitalisation [29-31], and/or death [32], and are not directly comparable to VE estimates against laboratory-confirmed influenza notifications as used in this study. A 2018 study of sentinel hospital site data in Australia found adjusted VE against any laboratory-confirmed influenza hospital admission in children to be $77 \%(95 \% \mathrm{Cl} 60-87)$ in children aged 6 months to $<2$ years, and $80 \%(95 \% \mathrm{Cl} 39-94)$ in children aged 2 years to $<5$ years [33].

Influenza VE was higher for children who were completely vaccinated in 2018 relative to those who were incompletely vaccinated — similar to findings in a recent meta-analysis for VE against influenzaassociated hospitalisation in children [34]. Our findings are also consistent with a recently published US test-negative design study among children aged 8 years or younger, where a higher VE was observed for children who received two doses in the current season compared to those who received one dose in the current season (and no prior doses) [35]. However, when stratified by age group, influenza vaccination had a statistically significant effect for children completely vaccinated only, with VE increasing with age and highest in children older than three years of age. Immunisation providers 
should adhere to the Australian Immunisation Handbook guidance regarding children receiving two doses in the first year of influenza vaccination to ensure maximum protection against seasonal influenza.

Influenza A subtype-specific VE estimates were limited due to incomplete subtype information, however our findings demonstrate a reasonably effective influenza vaccine in 2018-a year where circulating H3N2 (49\%) [36] was observed to be low when compared to previous years (2017: 83\%; 2016: 73\%) [10, 37]. Improving the availability of subtype information, particularly for high-risk age groups such as children, will allow for this assessment to be made routinely in the future. This is especially important for monitoring effectiveness against severe H3N2 infection which has been implicated in vaccine mismatch and reduced vaccine-preventability in recent years [38, 39].

A potential limitation of this study is underreporting of influenza vaccination to AIR. A recent study estimated the error rate of data transfer to AIR to be $14 \%$ nationally, and $8.5 \%$ in Queensland, with the most common reason for error related to the failure of transfer of vaccination encounter information from practice management software to AIR [40]. Our cases and controls had the same data source (AIR) for their vaccination status, though it is worth noting that influenza vaccination coverage estimates vary by data source. For example, coverage of any influenza vaccine among children was recorded as $29.4 \%$ among First Nations children and $25.1 \%$ among non-First Nations children aged 6 months to $<5$ years in Queensland in 2018 using AIR data [41]. This is higher than 17\% coverage reported in the same age group and year in Queensland when using a large general practitioner database [42]. In using AIR to estimate VE trends over time, potential bias due to misclassification of vaccination status is likely to be non-differential with respect to influenza outcome status, and should therefore lead to conservative VE estimates.

Vaccination coverage in the AIR among children aged 6 months to $<5$ years increased from $25.6 \%$ in 2018, to $39.7 \%$ in 2019 [43]. Following recommendation from the Australian Pharmaceutical Benefits Advisory Committee [44], influenza vaccination for this age group was funded under the National 
Immunisation Program (NIP) from 01 April 2020 [45]. With likely higher vaccination coverage as a result of funding under the NIP, and upgrades in practice management software systems, it is expected that reporting of vaccine encounters to the AIR by vaccine providers will also increase. With improvements in reporting to AIR comes the opportunity to develop more sophisticated mechanisms to routinely monitor the impact of the funded program, and, ideally, to estimate the indirect impact on influenza in older age groups. A suitable model for this would be to use the AIR and routinely collected jurisdictional influenza notification data, as demonstrated in this study.

As with most observational studies assessing VE, access to care and testing, and health-seeking behaviour are potential confounders that were unable to be assessed in this study. Australians have ready access to a government funded universal healthcare system, Medicare. In Australia, diagnostic testing for influenza is publicly funded when testing is required for clinical management [46], but there are no formal guidelines for influenza testing in children, with testing entirely at the discretion of the treating physician. PCR is widely available in Australia through public and private laboratories, and is now the dominant testing method for influenza-including in the age-group of children in this study [47]. Existing syndromic surveillance systems for influenza-like-illness in Australia are largely adultfocused, hence the need for specific systems to monitor VE in children $[48,49]$. Another potential confounder is testing based on vaccination status, but evidence in the literature on this topic is scarce. However, where data are available, they suggest testing based on vaccination status is unlikely to have any more than a minimal impact on VE estimates [50].

In our population, the southern hemisphere 2018 quadrivalent influenza vaccine provided moderate protection against laboratory confirmed infection in pre-school aged children. Effectiveness improved with increasing age and when national guidelines for repeat dosing in the first year of vaccination were followed. Further, our study also demonstrates the feasibility of VE estimates to be produced at a jurisdictional-level using routinely collected influenza notification and AIR data. Monitoring vaccine 
effectiveness at the community level, particularly for groups receiving funded vaccine, should be incorporated into Australia's routine surveillance of influenza and its impact.

\section{Declaration of Competing Interest}

The authors declare that they have no known competing financial interests or personal relationships that could have appeared to influence the work reported in this paper.

\section{Acknowledgements}

We thank the Queensland Health Communicable Diseases Branch Epidemiology and Research Unit, and Alexandra Hendry and Brynley Hull at the National Centre for Immunisation Research and Surveillance and the Australian Department of Health, for extracting and providing relevant data to complete this important work. Whilst doing this work Dharshi Thangarajah was supported by the Australian National University Master of Philosophy (Applied Epidemiology) Scholarship. 


\section{References}

[1] Australian Institute of Health and Welfare. The burden of vaccine preventable diseases in Australia. 2019. https://www.aihw.gov.au/getmedia/49809836-8ead-4da5-81c4-

352fa64df75b/aihw-phe-263.pdf.aspx?inline=true [accessed July 30, 2020] Cat. no. PHE 263.

[2] Department of Health. National Notifiable Diseases Surveillance System (NNDSS) data, influenza (laboratory confirmed). http://www9.health.gov.au/cda/source/rpt 4.cfm [accessed July 30, 2020].

[3] Li-Kim-Moy J, Yin JK, Patel C, Beard FH, Chiu C, Macartney KK, et al. Australian vaccine preventable disease epidemiological review series: Influenza 2006 to 2015. Commun Dis Intell Q Rep. 2016;40:E482-E95.

[4] Eichner M, Schwehm M, Eichner L, Gerlier L. Direct and indirect effects of influenza vaccination. BMC Infect Dis. 2017;17:308. https://doi.org/10.1186/s12879-017-2399-4

[5] Weidemann F, Remschmidt C, Buda S, Buchholz U, Ultsch B, Wichmann O. Is the impact of childhood influenza vaccination less than expected: a transmission modelling study. BMC Infect Dis. 2017;17:258. https://doi.org/10.1186/s12879-017-2344-6

[6] Guo D, Li KC, Peters TR, Snively BM, Poehling KA, Zhou X. Multi-scale modeling for the transmission of influenza and the evaluation of interventions toward it. Sci Rep. 2015;5:8980. https://doi.org/10.1038/srep08980

[7] Reichert TA, Sugaya N, Fedson DS, Glezen WP, Simonsen L, Tashiro M. The Japanese experience with vaccinating schoolchildren against influenza. N Engl J Med. 2001;344:889-96. https://doi.org/10.1056/NEJM200103223441204

[8] Loeb M, Russell ML, Moss L, Fonseca K, Fox J, Earn DJ, et al. Effect of influenza vaccination of children on infection rates in Hutterite communities: a randomized trial. JAMA. 2010;303:943-50. https://doi.org/10.1001/iama.2010.250

[9] Hodgson D, Baguelin M, van Leeuwen E, Panovska-Griffiths J, Ramsay M, Pebody R, et al. Effect of mass paediatric influenza vaccination on existing influenza vaccination programmes in England and Wales: a modelling and cost-effectiveness analysis. Lancet Public Health. 2017;2:e74-e81. https://doi.org/10.1016/S2468-2667(16)30044-5

[10] Queensland Health. Statewide Weekly Influenza Surveillance Report. Reporting Period: 1 January to 31 December 2017.

https://www.health.qld.gov.au/ data/assets/pdf file/0034/656494/influenza-qld-2017.pdf [accessed July 30, 2020].

[11] Queensland Health. Immunisation Program Update 2018 Influenza Special Edition. 2018. https://www.health.qld.gov.au/ data/assets/pdf file/0029/704891/qhip-news-18iss11.pdf [accessed July 30, 2020].

[12] Department of Health. Australian Technical Advisory Group on Immunisation (ATAGI) advice on seasonal influenza vaccines in 2018.

https://www.health.gov.au/sites/default/files/atagi clinical advice factsheet -

2018 influenza statement.pdf [accessed July 30, 2020].

[13] Department of Health. Australian Technical Advisory Group on Immunisation (ATAGI). Australian Immunisation Handbook. https://immunisationhandbook.health.gov.au/ [accessed July 30, 2020]. 
[14] Queensland Government. Public Health Act 2005.

https://www.legislation.qld.gov.au/view/pdf/inforce/current/act-2005-048 [accessed July 30, 2020].

[15] Queensland Government. Public Health Regulation 2018.

https://www.legislation.qld.gov.au/view/pdf/inforce/current/sl-2018-0117 [accessed July 30, 2020].

[16] Queensland Health. Queensland Notification Criteria. Guidelines for Laboratories. 2015.

https://www.health.qld.gov.au/ data/assets/pdf file/0034/429694/notif-criteria-guide.pdf

[accessed July 05, 2020].

[17] Department of Health. Seasonal Influenza Infection. CDNA National Guidelines for Public Health Units. 2017.

https://www1.health.gov.au/internet/main/publishing.nsf/Content/3D622AEAE44DDEB2CA257BF00 01ED884/\$SFile/influenza-infection-2019.pdf [accessed July 10, 2020].

[18] Department of Health. Australia's notifiable diseases status, 2009: Annual report of the National Notifiable Diseases Surveillance System - Notes. 2009.

https://www1.health.gov.au/internet/main/publishing.nsf/Content/cda-cdi3502a3.htm [accessed July 14, 2020].

[19] Services Australia. Australian Immunisation Register.

https://www.servicesaustralia.gov.au/individuals/services/medicare/australian-immunisation-

register [accessed July 30, 2020].

[20] Australian Bureau of Statistics. 1270.0.55.001 - Australian Statistical Geography Standard (ASGS): Volume 1 - Main Structure and Greater Capital City Statistical Areas, July 20162016. https://www.abs.gov.au/ausstats/abs@.nsf/Lookup/by\%20Subject/1270.0.55.001 July\%202016 M ain\%20Features Statistical\%20Area\%20Level\%202\%20(SA2) 10014 [accessed September 20, 2019].

[21] Australian Government. ASGS Correspondences (2016) - 2016 Population Weighted. 2018. https://data.gov.au/data/dataset/asgs-geographic-correspondences-2016/resource/951e18c7-f1874c86-a73f-fcabcd19af16 [accessed September 20, 2019].

[22] Queensland Government Statistician's Office. Meshblock correspondence file (ASGS 2016). 2016. https://www.qgso.qld.gov.au/geographies-maps/geographic-hierarchy/meshblockcorrespondence-file-asgs-2016 [accessed September 20, 2019].

[23] Australian Bureau of Statistics. Technical Paper, Socio-Economic Indexes for Areas (SEIFA) 2018. 2033.0.55.001

https://www.ausstats.abs.gov.au/ausstats/subscriber.nsf/0/756EE3DBEFA869EFCA258259000BA746 \\$SFile/SEIFA\%202016\%20Technical\%20Paper.pdf [accessed July 30, 2020].

[24] Australian Bureau of Statistics. The Australian Statistical Geography Standard (ASGS)

Remoteness Structure.

https://www.abs.gov.au/websitedbs/D3310114.nsf/home/remoteness+structure [accessed July 30, 2020].

[25] Department of Health. 2018 Influenza Season in Australia. A summary from the National Influenza Surveillance Committee. 2018.

https://www1.health.gov.au/internet/main/publishing.nsf/Content/CA086525758664B4CA2583620 0807AF9/\$File/2018-Season-Summary.pdf [accessed July 15, 2020].

[26] Powell LN, Begue RE. Influenza vaccine effectiveness among children for the 2017-2018 season. J Pediatric Infect Dis Soc. 2019 [In press]. https://doi.org/10.1093/ipids/piz077 
[27] Rolfes MA, Flannery B, Chung JR, O'Halloran A, Garg S, Belongia EA, et al. Effects of influenza vaccination in the United States during the 2017-2018 influenza season. Clin Infect Dis.

2019;69:1845-53. https://doi.org/10.1093/cid/ciz075

[28] Sullivan SG, Chilver MB, Carville KS, Deng YM, Grant KA, Higgins G, et al. Low interim influenza vaccine effectiveness, Australia, 1 May to 24 September 2017. Euro Surveill. 2017;22.

https://doi.org/10.2807/1560-7917.ES.2017.22.43.17-00707

[29] Blyth CC, Cheng AC, Finucane C, Jacoby P, Effler PV, Smith DW, et al. The effectiveness of influenza vaccination in preventing hospitalisation in children in Western Australia. Vaccine. 2015;33:7239-44. https://doi.org/10.1016/j.vaccine.2015.10.122

[30] Blyth CC, Macartney KK, Hewagama S, Senenayake S, Friedman ND, Simpson G, et al. Influenza epidemiology, vaccine coverage and vaccine effectiveness in children admitted to sentinel Australian hospitals in 2014: the Influenza Complications Alert Network (FluCAN). Euro Surveill. 2016;21. https://doi.org/10.2807/1560-7917.ES.2016.21.30.30301

[31] Blyth CC, Macartney KK, McRae J, Clark JE, Marshall HS, Buttery J, et al. Influenza epidemiology, vaccine coverage and vaccine effectiveness in children admitted to sentinel Australian hospitals in 2017: results from the PAEDS-FluCAN collaboration. Clin Infect Dis. 2019;68:940-8. https://doi.org/10.1093/cid/ciy597

[32] Nation ML, Moss R, Spittal MJ, Kotsimbos T, Kelly PM, Cheng AC. Influenza vaccine effectiveness against influenza-related mortality in Australian hospitalized patients: a propensity score analysis. Clin Infect Dis. 2020. https://doi.org/10.1093/cid/ciz1238

[33] Blyth CC, Cheng AC, Crawford NW, Clark JE, Buttery JP, Marshall HS, et al. The impact of new universal child influenza programs in Australia: Vaccine coverage, effectiveness and disease epidemiology in hospitalised children in 2018. Vaccine. 2020;38:2779-87.

https://doi.org/10.1016/i.vaccine.2020.02.031

[34] Kalligeros M, Shehadeh F, Mylona EK, Dapaah-Afriyie C, van Aalst R, Chit A, et al. Influenza vaccine effectiveness against influenza-associated hospitalization in children: A systematic review and meta-analysis. Vaccine. 2020;38:2893-903. https://doi.org/10.1016/j.vaccine.2020.02.049

[35] Chung JR, Flannery B, Gaglani M, Smith ME, Reis EC, Hickey RW, et al. Patterns of Influenza Vaccination and Vaccine Effectiveness Among Young US Children Who Receive Outpatient Care for Acute Respiratory Tract IIIness. JAMA Pediatr. 2020.

https://doi.org/10.1001/jamapediatrics.2020.0372

[36] Queensland Health. Statewide Influenza Surveillance Report. Reporting Period: 1 January to 31 December 2018. https://www.health.qld.gov.au/ data/assets/pdf file/0033/707649/influenzagld-2018.pdf [accessed July 30, 2020].

[37] Queensland Health. Statewide Weekly Influenza Surveillance Report. Reporting Period: 1 January to 31 December 2016.

https://www.health.qld.gov.au/ data/assets/pdf file/0030/444495/influenza-qld-2016.pdf [accessed July 30, 2020].

[38] Monto AS, Petrie JG. Improving Influenza Vaccine Effectiveness: Ways to Begin Solving the Problem. Clin Infect Dis. 2019;69:1824-6. https://doi.org/10.1093/cid/ciz416

[39] Belongia EA, McLean HQ. Influenza Vaccine Effectiveness: Defining the H3N2 Problem. Clin Infect Dis. 2019;69:1817-23. https://doi.org/10.1093/cid/ciz411 
[40] National Centre for Immunisation Research and Surveillance. Australian Immunisation Register Data Transfer Study Stage 2 Final Report. August 2018.

http://www.ncirs.org.au/sites/default/files/2018-

12/2018\%20AIR\%20data\%20tranfer\%20report FINAL 0.pdf [accessed July 30, 2020].

[41] National Centre for Immunisation Research and Surveillance. Annual Immunisation Coverage Report 2018 November 2018. http://ncirs.org.au/sites/default/files/2019-

11/NCIRS\%20Annual\%20Immunisation\%20Coverage\%20Report\%202018.pdf [accessed July 30, 2020].

[42] De Oliveira Bernardo C, Gonzalez-Chica DA, Stocks N. Impact of funding influenza vaccination on coverage among Australian children: a national study using Medicinelnsight, a large general practice database. Hum Vaccin Immunother. 2020;16:630-5.

https://doi.org/10.1080/21645515.2019.1664866

[43] Beard F, Hendry A, Macartney K. Influenza vaccination uptake in our most vulnerable groups: how well are we protecting them in 2019? Commun Dis Intell (2018). 2020;44.

https://doi.org/10.33321/cdi.2020.44.27

[44] Department of Health. July 2019 - PBAC meeting - positive recommendations.

Recommendations made by the PBAC - July 20192019.

http://www.pbs.gov.au/industry/listing/elements/pbac-meetings/pbac-outcomes/2019-07/positiverecommendations-07-2019.pdf [accessed July 30, 2020].

[45] Department of Health. National Immunisation Program Schedule. 2020.

https://www.health.gov.au/health-topics/immunisation/immunisation-throughout-life/nationalimmunisation-program-schedule\%E3\#national-immunisation-program-schedule-from-1-april-2019 [accessed July 30, 2020].

[46] Department of Health. Pathology - The Facts. How are pathology test fees calculated? https://www1.health.gov.au/internet/publications/publishing.nsf/Content/qupp-benefits-and-risksfor-consumers-of-pathology-testing qupp-fees [accessed July 22, 2020].

[47] Kaczmarek MC, Ware RS, Lambert SB. The contribution of PCR testing to influenza and pertussis notifications in Australia. Epidemiol Infect. 2016;144:306-14.

https://doi.org/10.1017/s0950268815001004

[48] Australian Sentinel Practices Research Network (ASPREN).

https://aspren.dmac.adelaide.edu.au/ [accessed July 14, 2020].

[49] FluTracking. FluTracking Reports (Australia). https://info.flutracking.net/reports-2/australiareports/ [accessed July 14, 2020].

[50] Ferdinands JM, Belongia EA, Nwasike C, Shay DK. Influenza vaccination status is not associated with influenza testing among children: implications for observational studies of vaccine effectiveness. Vaccine. 2011;29:1935-40. https://doi.org/10.1016/j.vaccine.2010.12.098 
Tables 
Table 1: Characteristics of influenza cases and matched controls included in this study, Queensland, 2018

\begin{tabular}{|c|c|c|c|}
\hline & Cases n (\%) & Controls n (\%) & $p$-value \\
\hline \multicolumn{4}{|l|}{ Age at case onseta } \\
\hline $6 \mathrm{~m} 14 \mathrm{~d}$ to $<2 y$ & $283(25.2)$ & $2,647(25.0)$ & \multirow{4}{*}{1.00} \\
\hline $2 y$ to $<3 y$ & $213(18.9)$ & 2,007 (18.8) & \\
\hline $3 y$ to $<4 y$ & $230(20.4)$ & $2,196(20.6)$ & \\
\hline $4 y$ to $<6 y$ & $399(35.5)$ & $3,795(35.6)$ & \\
\hline \multicolumn{4}{|l|}{ Sex } \\
\hline Male & $588(52.3)$ & $5,544(52.1)$ & \multirow{2}{*}{0.9} \\
\hline Female & $537(47.7)$ & $5,101(47.9)$ & \\
\hline \multicolumn{4}{|l|}{ First Nations status } \\
\hline First Nations child & $105(9.3)$ & $807(7.6)$ & \multirow{2}{*}{0.04} \\
\hline Non-First Nations child & $1,020(90.7)$ & $9,838(92.4)$ & \\
\hline \multicolumn{4}{|l|}{ Remoteness area } \\
\hline Major City & $767(68.2)$ & $7,348(69.0)$ & \multirow{5}{*}{0.91} \\
\hline Inner regional & $166(14.8)$ & $1,513(14.2)$ & \\
\hline Outer regional & $181(16.1)$ & $1,698(16.0)$ & \\
\hline Remote & $5(0.4)$ & $46(0.4)$ & \\
\hline Very remote & $6(0.5)$ & $40(0.4)$ & \\
\hline \multicolumn{4}{|l|}{ IRSAD quintile } \\
\hline 1 st $(0 \%$ to $<20 \%)$ & $248(22.0)$ & $2,408(22.6)$ & \multirow{5}{*}{0.94} \\
\hline $2^{\text {nd }}(20 \%$ to $<40 \%)$ & $145(12.9)$ & $1,315(12.4)$ & \\
\hline $3^{\text {rd }}(40 \%$ to $<60 \%)$ & $251(22.3)$ & $2,337(22.0)$ & \\
\hline $4^{\text {th }}(60 \%$ to $<80 \%)$ & $279(24.8)$ & $2,724(25.6)$ & \\
\hline $5^{\text {th }}(80 \%$ to $100 \%)$ & $202(18.0)$ & $1,861(17.5)$ & \\
\hline Total & $1,125(100)$ & $10,645(100)$ & \\
\hline
\end{tabular}

Abbreviations: $d$, days; m, months; $y$, years; IRSAD, Index of Relative Socio-economic Advantage and

Disadvantage.

Note: Association between characteristic and case-control status assessed using the chi-squared test

${ }^{a}$ Age at onset of case. For controls: age of control at onset of matched case. 
Table 2: Number of cases and controls, and VE estimates by vaccination history among children eligible for the funded influenza vaccine, Queensland, 2018

\begin{tabular}{|c|c|c|c|c|}
\hline & Cases n (\%) & Controls n (\%) & Crude VE $(95 \% \mathrm{Cl})$ & Adjusteda VE $(95 \% \mathrm{Cl})$ \\
\hline \multicolumn{5}{|l|}{ Vaccinated (any) } \\
\hline No & $991(88.1)$ & $8,369(78.6)$ & Ref. & Ref. \\
\hline Yes & $134(11.9)$ & $2,276(21.4)$ & $51(41-59)$ & $51(41-60)$ \\
\hline \multicolumn{5}{|l|}{ Vaccine doses } \\
\hline No vaccination & $991(88.1)$ & $8,369(78.6)$ & Ref. & Ref. \\
\hline 1 & $80(7.1)$ & $1,172(11.0)$ & $42(27-55)$ & $43(28-55)$ \\
\hline 2 & $54(4.8)$ & $1,104(10.4)$ & $60(47-70)$ & $60(46-70)$ \\
\hline \multicolumn{5}{|c|}{ Current and prior year vaccine dose receipt } \\
\hline No vaccination & $991(88.1)$ & $8,369(78.6)$ & Ref. & Ref. \\
\hline 1 dose (no prior dose) & $62(5.5)$ & $806(7.6)$ & $35(15-50)$ & $35(15-51)$ \\
\hline 1 dose (prior dose) & $18(1.6)$ & $366(3.4)$ & $59(34-75)$ & $60(35-75)$ \\
\hline 2 doses & $54(4.8)$ & $1,104(10.4)$ & $60(47-70)$ & $60(46-70)$ \\
\hline \multicolumn{5}{|c|}{ Vaccination completeness $^{b}$} \\
\hline No vaccination & $991(88.1)$ & $8,369(78.6)$ & Ref. & Ref. \\
\hline Incomplete vaccination & $62(5.5)$ & $806(7.6)$ & $35(15-50)$ & $35(15-51)$ \\
\hline Complete vaccination & $72(6.4)$ & $1,470(13.8)$ & 60 (48-69) & $60(48-69)$ \\
\hline
\end{tabular}

Abbreviations: VE, vaccine effectiveness; Ref., reference group.

${ }^{a}$ Adjusted for sex and First Nations status.

baccination completeness: incomplete vaccination, one dose in 2018 and no prior dose; complete vaccination, one dose in 2018 and at least one prior dose, or two doses in 2018. 
Table 3: Number of cases and controls, and age-stratified VE estimates by vaccination history among children eligible for the funded influenza vaccine, Queensland, 2018

\begin{tabular}{|c|c|c|c|c|}
\hline & Cases n (\%) & Controls n (\%) & Crude VE $(95 \% \mathrm{Cl})$ & Adjusted $^{a}$ VE $(95 \% \mathrm{Cl})$ \\
\hline \multicolumn{5}{|c|}{ Vaccination completeness stratified by age group } \\
\hline \multicolumn{5}{|l|}{$6 \mathrm{~m} 14 \mathrm{~d}$ to $<2 \mathrm{y}$} \\
\hline No vaccination & $236(83.4)$ & $2,043(77.0)$ & Ref. & Ref. \\
\hline Any vaccination & $47(16.1)$ & $615(23.0)$ & $33(7-52)$ & $33(7-52)$ \\
\hline Incomplete vaccinationc & $22(7.8)$ & $248(9.3)$ & $21(-25-50)$ & $24(-22-52)$ \\
\hline Complete vaccination ${ }^{d}$ & $25(8.8)$ & $367(13.8)$ & $41(9-62)$ & $40(7-61)$ \\
\hline \multicolumn{5}{|l|}{$2 y$ to $<3 y$} \\
\hline No vaccination & $184(86.4)$ & $1,532(76.7)$ & Ref. & Ref. \\
\hline Any vaccination & $29(13.6)$ & $466(23.3)$ & $48(21-65)$ & $48(21-65)$ \\
\hline Incomplete vaccinationc & $8(3.8)$ & $154(7.7)$ & $56(9-79)$ & $56(9-79)$ \\
\hline Complete vaccination ${ }^{d}$ & $21(9.9)$ & $312(15.6)$ & $44(9-65)$ & $44(10-65)$ \\
\hline \multicolumn{5}{|l|}{$3 y$ to $<4 y$} \\
\hline No vaccination & $213(92.6)$ & $1,745(80.1)$ & Ref. & Ref. \\
\hline Any vaccination & $17(7.4)$ & $434(19.9)$ & $69(48-81)$ & $69(48-81)$ \\
\hline Incomplete vaccinationc & $8(3.5)$ & $131(6.0)$ & $51(-1-77)$ & $52(0-77)$ \\
\hline Complete vaccination ${ }^{d}$ & $9(3.9)$ & $303(13.9)$ & $76(53-88)$ & 77 (54-88) \\
\hline \multicolumn{5}{|l|}{$4 y$ to $<6 y$} \\
\hline No vaccination & $358(89.7)$ & $3,049(80.0)$ & Ref. & Ref. \\
\hline Any vaccination & $41(10.3)$ & $761(20.0)$ & $56(38-68)$ & $56(38-68)$ \\
\hline Incomplete vaccination ${ }^{c}$ & $24(6.0)$ & $273(7.2)$ & $25(-16-52)$ & $25(-15-52)$ \\
\hline Complete vaccination ${ }^{d}$ & $17(4.3)$ & $488(12.8)$ & $72(54-83)$ & $72(54-83)$ \\
\hline
\end{tabular}

Abbreviations: VE, vaccine effectiveness; Ref., reference group; $d$, days; $m$, months; $y$, years.

${ }^{a}$ Adjusted for sex and First Nations status.

${ }^{\mathrm{b}}$ For controls, age is at onset of matched case

Incomplete vaccination: One dose in 2018 and no prior dose.

${ }^{\mathrm{d} C}$ Complete vaccination: One dose in 2018 and at least one prior dose, or two doses in 2018. 
Table 4: Number of cases and controls, and influenza subtype vaccine effectiveness estimates by vaccination history among children eligible for the funded influenza vaccine, Queensland, 2018

\begin{tabular}{|c|c|c|c|c|}
\hline & Cases n (\%) & Controls n (\%) & Crude VE $(95 \% \mathrm{CI})$ & Adjusted $^{a}$ VE $(95 \% \mathrm{Cl})$ \\
\hline \multicolumn{5}{|l|}{ Vaccine doses } \\
\hline \multicolumn{5}{|l|}{ Influenza A (all) } \\
\hline No vaccination & $939(88.1)$ & $7,916(78.6)$ & Ref. & Ref. \\
\hline Vaccination (any) & $127(11.9)$ & $2,161(21.4)$ & $51(41-60)$ & $51(41-60)$ \\
\hline 1 dose & $75(7.1)$ & $1,100(10.9)$ & $43(27-55)$ & $44(28-56)$ \\
\hline 2 doses & $52(4.9)$ & $1,061(10.5)$ & $60(46-70)$ & $60(46-70)$ \\
\hline \multicolumn{5}{|l|}{ Influenza A H1N1 } \\
\hline No vaccination & $122(89.1)$ & $1,043(78.8)$ & Ref. & Ref. \\
\hline Vaccination (any) & $15(11.0)$ & $281(21.2)$ & $54(20-74)$ & $54(20-74)$ \\
\hline 1 dose & $11(8.0)$ & $138(10.4)$ & $31(-31-64)$ & $33(-28-65)$ \\
\hline 2 doses & $4(2.9)$ & $143(10.8)$ & 77 (35-92) & $76(33-91)$ \\
\hline \multicolumn{5}{|l|}{ Influenza A H3N2 } \\
\hline No vaccination & $45(84.9)$ & $404(77.7)$ & Ref. & Ref. \\
\hline Vaccination (any) & $8(15.1)$ & $116(22.3)$ & $39(-33-72)$ & $40(-32-73)$ \\
\hline 1 dose & $3(5.7)$ & $62(11.9)$ & $57(-44-87)$ & $58(-41-87)$ \\
\hline 2 doses & $5(9.4)$ & $54(10.4)$ & $20(-111-70)$ & $19(-114-70)$ \\
\hline \multicolumn{5}{|l|}{ Influenza B } \\
\hline No vaccination & $52(88.1)$ & $453(79.8)$ & Ref. & Ref. \\
\hline Vaccination (any) & $7(11.9)$ & $115(20.2)$ & $46(-22-76)$ & $44(-27-75)$ \\
\hline 1 dose & $5(8.5)$ & $72(12.7)$ & $39(-60-76)$ & $36(-68-75)$ \\
\hline 2 doses & $2(3.4)$ & $43(7.6)$ & $59(-78-90)$ & $57(-84-90)$ \\
\hline \multicolumn{5}{|l|}{ Vaccine completeness } \\
\hline \multicolumn{5}{|l|}{ Influenza A (all) } \\
\hline No vaccination & $939(88.1)$ & $7,916(78.6)$ & Ref. & Ref. \\
\hline Incomplete vaccination ${ }^{b}$ & $58(5.4)$ & $750(7.4)$ & $35(14-50)$ & $35(14-51)$ \\
\hline Complete vaccination $^{c}$ & $69(6.5)$ & $1,411(14.0)$ & $60(48-69)$ & $60(48-69)$ \\
\hline \multicolumn{5}{|l|}{ Influenza A H1N1 } \\
\hline No vaccination & $122(89.1)$ & $1,043(78.8)$ & Ref. & Ref. \\
\hline Incomplete vaccination ${ }^{b}$ & $9(6.6)$ & $100(7.6)$ & $22(-58-62)$ & $25(-53-63)$ \\
\hline Complete vaccination $^{c}$ & $6(4.4)$ & $181(13.7)$ & $72(35-88)$ & $71(34-88)$ \\
\hline \multicolumn{5}{|l|}{ Influenza A H3N2 } \\
\hline No vaccination & $45(84.9)$ & $404(77.7)$ & Ref. & Ref. \\
\hline Incomplete vaccination ${ }^{b}$ & $2(3.8)$ & $43(8.3)$ & $58(-81-90)$ & $58(-79-90)$ \\
\hline
\end{tabular}




\begin{tabular}{lllll}
\hline & Cases n (\%) & Controls n (\%) & Crude VE (95\% CI) & Adjusted $^{\text {V VE }(95 \% \text { CI) }}$ \\
\hline Complete vaccination $^{c}$ & $6(11.3)$ & $73(14.0)$ & $29(-74-71)$ & $29(-73-71)$ \\
Influenza B & & & & \\
\hline No vaccination & $52(88.1)$ & $453(79.8)$ & Ref. & Ref. \\
Incomplete vaccination $^{b}$ & $4(6.8)$ & $56(9.8)$ & $37(-83-78)$ & $34(-92-77)$ \\
Complete vaccination $^{c}$ & $3(5.1)$ & $59(10.4)$ & $55(-51-87)$ & $53(-57-86)$ \\
\hline
\end{tabular}

Abbreviations: VE, vaccine effectiveness; Ref., reference group.

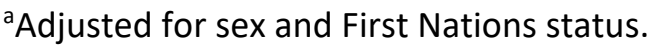

bIncomplete vaccination: One dose in 2018 and no prior dose.

'Complete vaccination: One dose in 2018 and at least one prior dose, or two doses in 2018. 
Figures 
Figure 1: Participant flow diagram of case and control enrolment and matching. Abbreviations:

NoCS, Queensland Notifiable Conditions System; AIR, Australian Immunisation Register; SA2,

Statistical Area 2; DOB, date of birth.

Enrolment

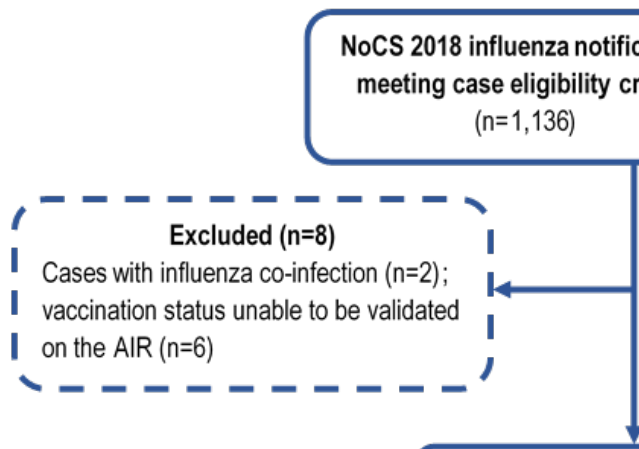

Cases eligible for matchin $(n=1,128)$

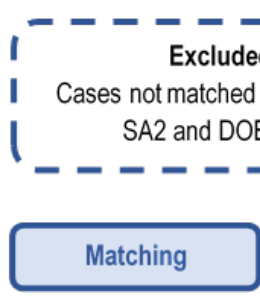

Control assignment
CASES

CONTROLS

AIR records of Queensland resident children meeting control eligibility criteria $(n=333,411)$
Controls randomly assigned to match to 1 case only ( 3,943 controls matched to $>1$ case $)$

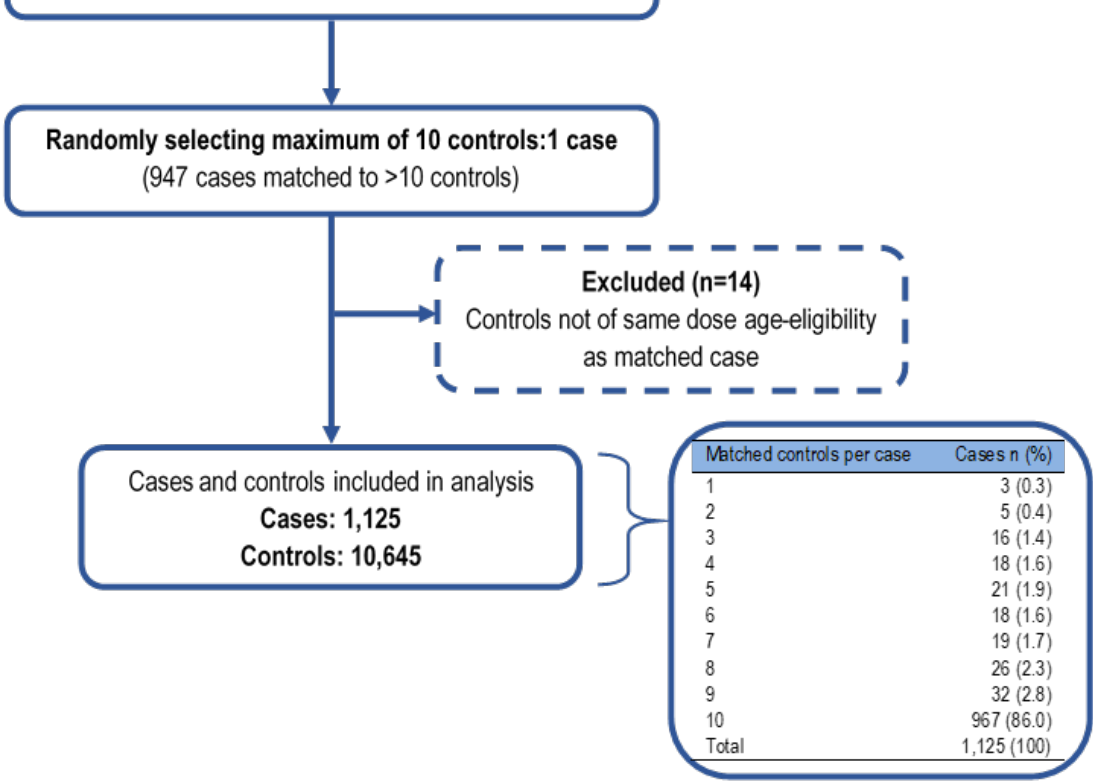


Figure 2: Receipt of influenza vaccine doses among study participants and influenza notifications among children aged 6 months and 14 days to $<5$ years,

Queensland, 2018. Note: Influenza notifications from May 2018 (week 18) to December 2018 (week 52 ) represent cases included in the study.

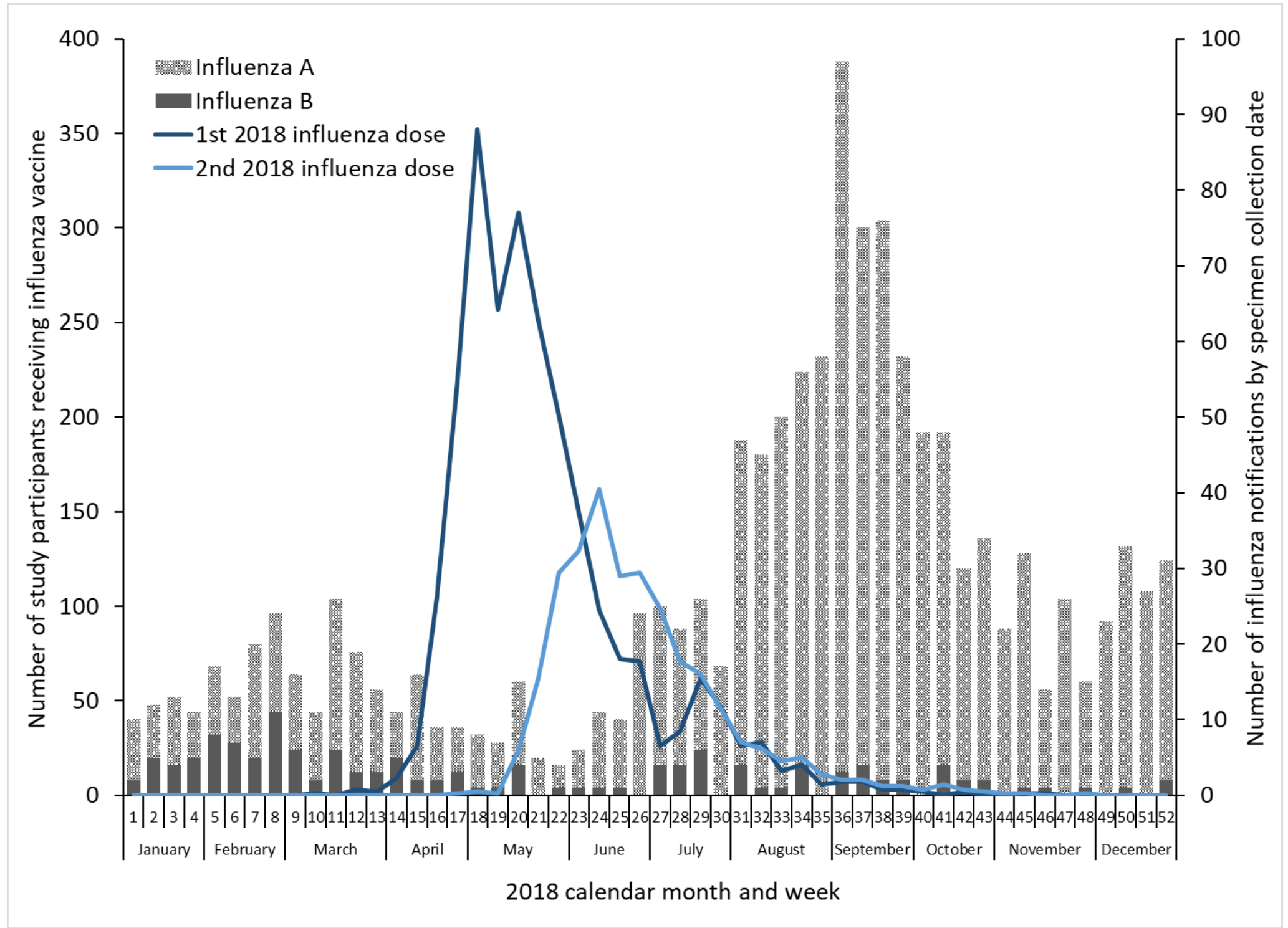

